\title{
ASYMPTOTIC EXPANSIONS FOR RATIOS OF PRODUCTS OF GAMMA FUNCTIONS
}

\author{
WOLFGANG BÜHRING
}

Received 6 May 2002

An asymptotic expansion for a ratio of products of gamma functions is derived.

2000 Mathematics Subject Classification: 33B15, 33C20.

1. Introduction. An asymptotic expansion for a ratio of products of gamma functions has recently been found [2], which, with

$$
s_{1}=b_{1}-a_{1}-a_{2},
$$

may be written as

$$
\frac{\Gamma\left(a_{1}+n\right) \Gamma\left(a_{2}+n\right)}{\Gamma\left(b_{1}+n\right) \Gamma\left(-s_{1}+n\right)}=1+\sum_{m=1}^{M} \frac{\left(s_{1}+a_{1}\right)_{m}\left(s_{1}+a_{2}\right)_{m}}{(1)_{m}\left(1+s_{1}-n\right)_{m}}+O\left(n^{-M-1}\right)
$$

as $n \rightarrow \infty$. Here, we make use of the Pochhammer symbol

$$
(x)_{n}=x(x+1) \cdots(x+n-1)=\Gamma(x+n) / \Gamma(x) .
$$

The special case when $b_{1}=1$ of formula (1.2) had been stated earlier by Dingle [3], and there were proofs by Paris [8] and Olver [6, 7].

The proof of (1.2) is based on the formula for the analytic continuation near unit argument of the Gaussian hypergeometric function. For the more general hypergeometric functions

$$
{ }_{p+1} F_{p}\left(\begin{array}{c}
a_{1}, a_{2}, \ldots, a_{p+1} \\
b_{1}, \ldots, b_{p}
\end{array} \mid z\right)=\sum_{n=0}^{\infty} \frac{\left(a_{1}\right)_{n}\left(a_{2}\right)_{n} \cdots\left(a_{p+1}\right)_{n}}{\left(b_{1}\right)_{n} \cdots\left(b_{p}\right)_{n}(1)_{n}} z^{n}, \quad(|z|<1)
$$

the analytic continuation near $z=1$ is known too, and this raises the question as to whether a sufficiently simple asymptotic expansion can be derived in a similar way for a ratio of products of more gamma function factors. This is indeed the case, and it is the purpose of this work to present such an expansion. 
2. Derivation of the asymptotic expansion. The analytic continuation of the hypergeometric function near unit argument may be written as (see [1])

$$
\begin{array}{r}
\frac{\Gamma\left(a_{1}\right) \Gamma\left(a_{2}\right) \cdots \Gamma\left(a_{p+1}\right)}{\Gamma\left(b_{1}\right) \cdots \Gamma\left(b_{p}\right)}{ }_{p+1} F_{p}\left(\begin{array}{c}
a_{1}, a_{2}, \ldots, a_{p+1} \\
b_{1}, \ldots, b_{p}
\end{array} \mid z\right) \\
=\sum_{m=0}^{\infty} g_{m}(0)(1-z)^{m}+(1-z)^{s_{p}} \sum_{m=0}^{\infty} g_{m}\left(s_{p}\right)(1-z)^{m},
\end{array}
$$

where

$$
s_{p}=b_{1}+\cdots+b_{p}-a_{1}-a_{2}-\cdots-a_{p+1}
$$

and the coefficients $g_{m}$ are known. While the $g_{m}(0)$ are not needed for the present purpose, the $g_{m}\left(s_{p}\right)$ are [1]

$$
\begin{aligned}
g_{m}\left(s_{p}\right)= & (-1)^{m} \frac{\left(a_{1}+s_{p}\right)_{m}\left(a_{2}+s_{p}\right)_{m} \Gamma\left(-s_{p}-m\right)}{(1)_{m}} \\
& \times \sum_{k=0}^{m} \frac{(-m)_{k}}{\left(a_{1}+s_{p}\right)_{k}\left(a_{2}+s_{p}\right)_{k}} A_{k}^{(p)},
\end{aligned}
$$

where the coefficients $A_{k}^{(p)}$ are to be shown below.

The left-hand side $L$ of (2.1) is

$$
L=\frac{\Gamma\left(a_{1}+n\right) \Gamma\left(a_{2}+n\right) \cdots \Gamma\left(a_{p+1}+n\right)}{\Gamma\left(b_{1}+n\right) \cdots \Gamma\left(b_{p}+n\right) \Gamma(1+n)} z^{n} .
$$

The asymptotic behaviour, as $n \rightarrow \infty$, of the coefficients of this power series is governed $[4,5,10]$ by the terms $R$ on the right-hand side which, at $z=1$, are singular

$$
R=\sum_{m=0}^{\infty} g_{m}\left(s_{p}\right)(1-z)^{s_{p}+m}
$$

Expanded by means of the binomial theorem in its hypergeometric-series form, this is

$$
R=\sum_{m=0}^{\infty} g_{m}\left(s_{p}\right) \sum_{n=0}^{\infty} \frac{\left(-s_{p}-m\right)_{n}}{\Gamma(1+n)} z^{n}
$$

Interchanging the order of summation (and making use of the reflection formula of the gamma function), we may get

$$
R=\sum_{n=0}^{\infty} \frac{\Gamma\left(-s_{p}+n\right)}{\Gamma(1+n)} \sum_{m=0}^{\infty}(-1)^{m} g_{m}\left(s_{p}\right) \frac{1}{\Gamma\left(-s_{p}-m\right)\left(1+s_{p}-n\right)_{m}} z^{n} .
$$


Comparison of the coefficients of the two power series for $R$ and $L$, which should agree asymptotically as $n \rightarrow \infty$, then leads to

$$
\begin{aligned}
& \frac{\Gamma\left(a_{1}+n\right) \Gamma\left(a_{2}+n\right) \cdots \Gamma\left(a_{p+1}+n\right)}{\Gamma\left(b_{1}+n\right) \cdots \Gamma\left(b_{p}+n\right) \Gamma\left(-s_{p}+n\right)} \\
& \sim \sum_{m=0}^{\infty}(-1)^{m} g_{m}\left(s_{p}\right) \frac{1}{\Gamma\left(-s_{p}-m\right)\left(1+s_{p}-n\right)_{m}} \text {. }
\end{aligned}
$$

Inserting $g_{m}$ from (2.3) and keeping the first $M+1$ terms of the asymptotic series, we get the following theorem.

THEOREM 2.1. With $s_{p}=b_{1}+\cdots+b_{p}-a_{1}-a_{2}-\cdots-a_{p+1}$, we have the asymptotic expansion

$$
\begin{aligned}
& \frac{\Gamma\left(a_{1}+n\right) \Gamma\left(a_{2}+n\right) \cdots \Gamma\left(a_{p+1}+n\right)}{\Gamma\left(b_{1}+n\right) \cdots \Gamma\left(b_{p}+n\right) \Gamma\left(-s_{p}+n\right)} \\
& \quad=1+\sum_{m=1}^{M} \frac{\left(a_{1}+s_{p}\right)_{m}\left(a_{2}+s_{p}\right)_{m}}{(1)_{m}\left(1+s_{p}-n\right)_{m}} \sum_{k=0}^{m} \frac{(-m)_{k}}{\left(a_{1}+s_{p}\right)_{k}\left(a_{2}+s_{p}\right)_{k}} A_{k}^{(p)}+O\left(n^{-M-1}\right)
\end{aligned}
$$

as $n \rightarrow \infty$.

The simple formula (1.2), corresponding to $p=1$, can be recovered from this theorem if we define $A_{0}^{(1)}=1, A_{k}^{(1)}=0$ for $k>0$, so that the sum over $k$ is then equal to 1 and disappears. The coefficients for larger $p$ can be found in [1], but a few of them are displayed again here for convenience

$$
\begin{aligned}
A_{k}^{(2)}= & \frac{\left(b_{2}-a_{3}\right)_{k}\left(b_{1}-a_{3}\right)_{k}}{k !}, \\
A_{k}^{(3)}= & \sum_{k_{2}=0}^{k} \frac{\left(b_{3}+b_{2}-a_{4}-a_{3}+k_{2}\right)_{k-k_{2}}\left(b_{1}-a_{3}\right)_{k-k_{2}}\left(b_{3}-a_{4}\right)_{k_{2}}\left(b_{2}-a_{4}\right)_{k_{2}}}{\left(k-k_{2}\right) ! k_{2} !} \\
A_{k}^{(4)}= & \sum_{k_{2}=0}^{k} \frac{\left(b_{4}+b_{3}+b_{2}-a_{5}-a_{4}-a_{3}+k_{2}\right)_{k-k_{2}}\left(b_{1}-a_{3}\right)_{k-k_{2}}}{\left(k-k_{2}\right) !} \\
& \times \sum_{k_{3}=0}^{k_{2}} \frac{\left(b_{4}+b_{3}-a_{5}-a_{4}+k_{3}\right)_{k_{2}-k_{3}}\left(b_{2}-a_{4}\right)_{k_{2}-k_{3}}}{\left(k_{2}-k_{3}\right) !} \frac{\left(b_{4}-a_{5}\right)_{k_{3}}\left(b_{3}-a_{5}\right)_{k_{3}}}{k_{3} !} .
\end{aligned}
$$

For $p=3,4, \ldots$, several other representations are possible [1] such as

$$
\begin{aligned}
A_{k}^{(3)}= & \frac{\left(b_{3}+b_{2}-a_{4}-a_{3}\right)_{k}\left(b_{1}-a_{3}\right)_{k}}{k !} \\
& \times{ }_{3} F_{2}\left(\begin{array}{c}
b_{3}-a_{4}, b_{2}-a_{4},-k \\
b_{3}+b_{2}-a_{4}-a_{3}, 1+a_{3}-b_{1}-k
\end{array} \mid \begin{array}{l}
1
\end{array}\right)
\end{aligned}
$$


or

$$
\begin{aligned}
& A_{k}^{(3)}=\frac{\left(b_{1}+b_{3}-a_{3}-a_{4}\right)_{k}\left(b_{2}+b_{3}-a_{3}-a_{4}\right)_{k}}{k !} \\
& \times_{3} F_{2}\left(\begin{array}{c}
b_{3}-a_{3}, b_{3}-a_{4},-k \\
b_{1}+b_{3}-a_{3}-a_{4}, b_{2}+b_{3}-a_{3}-a_{4}
\end{array} \mid 1\right) \text {. }
\end{aligned}
$$

For $p=2$, (2.9) may be simply written as

$$
\begin{aligned}
& \frac{\Gamma\left(a_{1}+n\right) \Gamma\left(a_{2}+n\right) \Gamma\left(a_{3}+n\right)}{\Gamma\left(b_{1}+n\right) \Gamma\left(b_{2}+n\right) \Gamma\left(-s_{2}+n\right)} \\
& \quad=1+\sum_{m=1}^{M} \frac{\left(a_{1}+s_{2}\right)_{m}\left(a_{2}+s_{2}\right)_{m}}{(1)_{m}\left(1+s_{2}-n\right)_{m}}{ }_{3} F_{2}\left(\begin{array}{c}
b_{2}-a_{3}, b_{1}-a_{3},-m \\
a_{1}+s_{2}, a_{2}+s_{2}
\end{array} \mid 1\right)+O\left(n^{-M-1}\right),
\end{aligned}
$$

where $s_{2}=b_{1}+b_{2}-a_{1}-a_{2}-a_{3}$.

3. Additional comments. The derivation of the theorem is based on the continuation formula (2.1) which holds, as it stands, only if $s_{p}$ is not equal to an integer. Nevertheless, the theorem is valid without such a restriction. This can be verified if the derivation is repeated starting from any of the continuation formulas for the exceptional cases [1]. Instead of or in addition to the binomial theorem, the expansion

$$
(1-z)^{m} \ln (1-z)=\sum_{n=1}^{\infty} c_{n} z^{n}
$$

is then needed for integer $m \geq 0$, where

$$
c_{n}=-\frac{1}{n}(-1)^{m} \frac{\Gamma(1+m) \Gamma(n-m)}{\Gamma(n)}
$$

for $n>m$, while the coefficients are not needed here for $n \leq m$.

The theorem has been proved here for any sufficiently large positive integer $n$ only. On the basis of the discussion in [2], it can be suspected that the theorem may be theoretically valid (although less useful) in the larger domain of the complex $n$-half-plane $\operatorname{Re}\left(s_{p}+a_{1}+a_{2}-1+n\right) \geq 0$.

Expansions for ratios of even more general products of gamma functions are treated in a recent monograph by Paris and Kaminski [9].

\section{REFERENCES}

[1] W. Bühring, Generalized hypergeometric functions at unit argument, Proc. Amer. Math. Soc. 114 (1992), no. 1, 145-153.

[2] - An asymptotic expansion for a ratio of products of gamma functions, Int. J. Math. Math. Sci. 24 (2000), no. 8, 505-510. 
[3] R. B. Dingle, Asymptotic Expansions: Their Derivation and Interpretation, Academic Press, London, 1973.

[4] P. Flajolet and A. Odlyzko, Singularity analysis of generating functions, SIAM J. Discrete Math. 3 (1990), no. 2, 216-240.

[5] F. W. J. Olver, Asymptotics and Special Functions, Computer Science and Applied Mathematics, Academic Press, New York, 1974.

[6] _ Asymptotic expansions of the coefficients in asymptotic series solutions of linear differential equations, Methods Appl. Anal. 1 (1994), no. 1, 1-13.

[7] _ On an asymptotic expansion of a ratio of gamma functions, Proc. Roy. Irish Acad. Sect. A 95 (1995), no. 1, 5-9.

[8] R. B. Paris, Smoothing of the Stokes phenomenon using Mellin-Barnes integrals, J. Comput. Appl. Math. 41 (1992), no. 1-2, 117-133.

[9] R. B. Paris and D. Kaminski, Asymptotics and Mellin-Barnes integrals, Encyclopedia of Mathematics and its Applications, vol. 85, Cambridge University Press, Cambridge, 2001.

[10] R. Schäfke and D. Schmidt, The connection problem for general linear ordinary differential equations at two regular singular points with applications in the theory of special functions, SIAM J. Math. Anal. 11 (1980), no. 5, 848-862.

Wolfgang Bühring: Physikalisches Institut, Universität Heidelberg, Philosophenweg 12, 69120 Heidelberg, Germany

E-mail address: buehring@physi .uni-heidelberg.de 


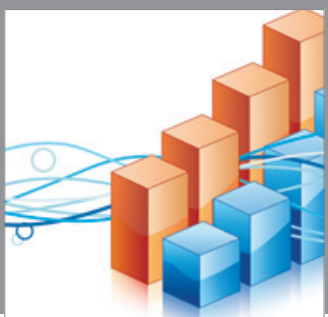

Advances in

Operations Research

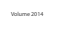

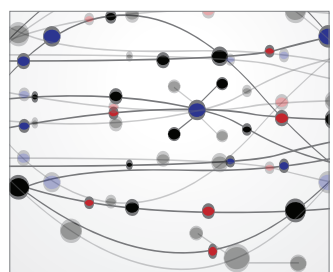

\section{The Scientific} World Journal
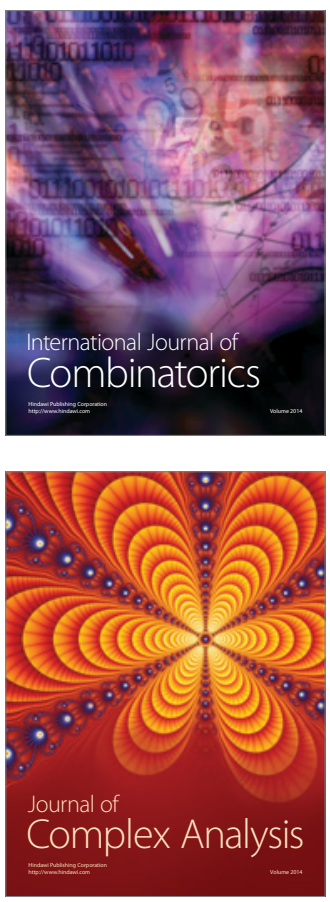

International Journal of

Mathematics and

Mathematical

Sciences
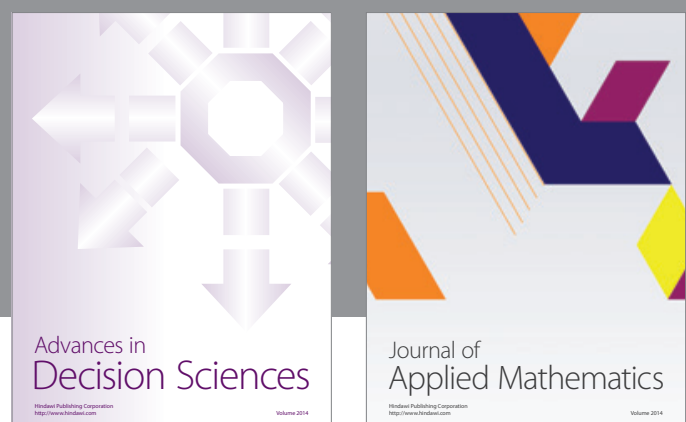

Journal of

Applied Mathematics
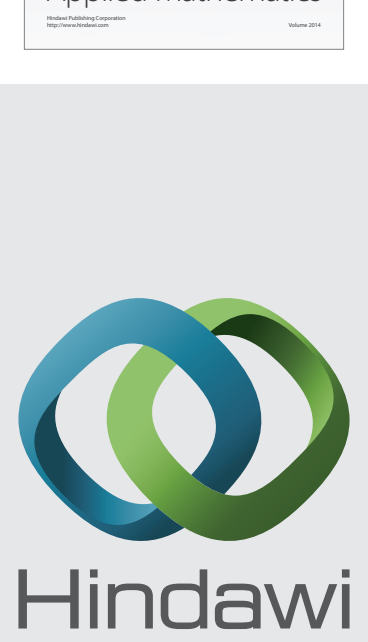

Submit your manuscripts at http://www.hindawi.com
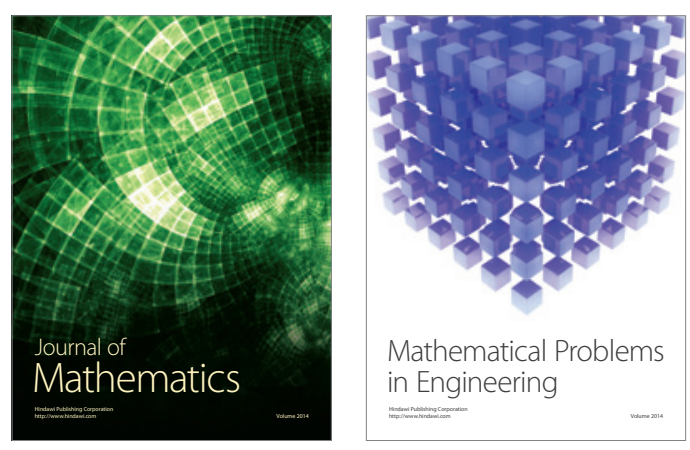

Mathematical Problems in Engineering
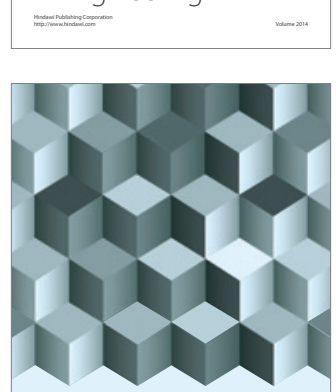

Journal of

Function Spaces
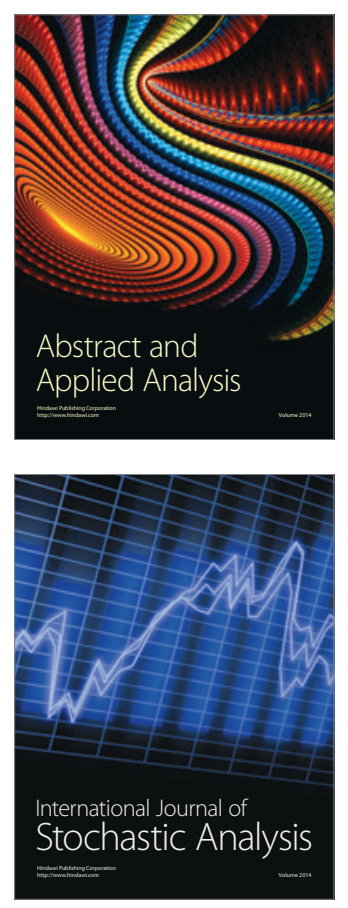

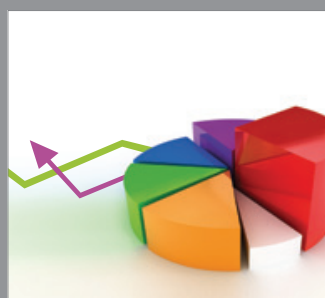

ournal of

Probability and Statistics

Promensencen
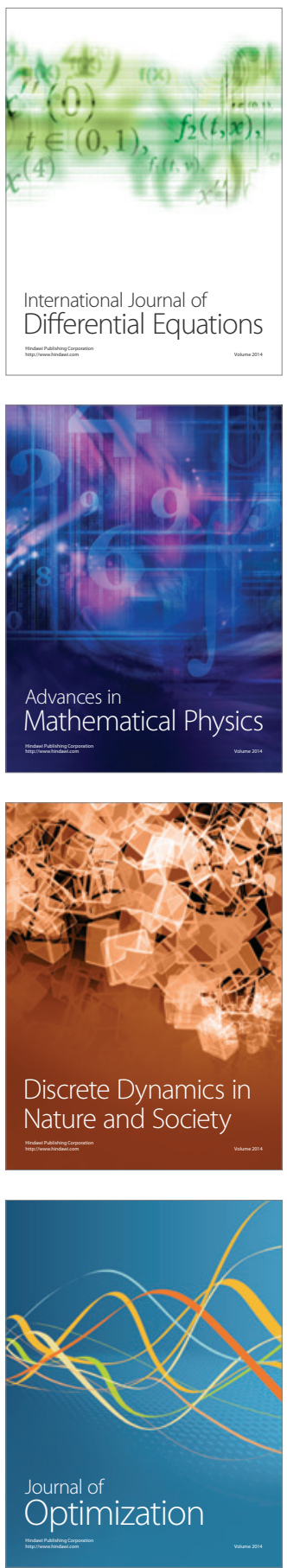